\title{
Gelatin sponge microparticles for the treatment of the spontaneous rupture of hepatocellular carcinoma hemorrhage
}

\author{
PU ZHAO WU, JUN ZHOU and YUE WEI ZHANG \\ Department of Interventional Therapy, Affiliated Zhongshan Hospital of Dalian University, \\ Dalian, Liaoning 116001, P.R. China
}

Received April 21, 2015; Accepted June 6, 2016

DOI: $10.3892 /$ etm.2016.3573

\begin{abstract}
Spontaneous rupture of hepatocellular carcinoma hemorrhage is life-threatening. The aim of the present study was to retrospectively analyze the effect of gelatin sponge microparticles (GSMs) of various diameters on the treatment of spontaneous rupture of hepatocellular carcinoma hemorrhage. GSMs serve as embolization agents by transcatheter arterial chemoembolization (TACE), and the current study analyzed their safety and efficacy. Data from a total of 13 cases of spontaneous rupture of hepatocellular carcinoma hemorrhage, who were treated with GSM-TACE at the Affiliated Zhongshan Hospital of Dalian University (Dalian, China) between August 2010 and June 2014, were collected. Post-operative complications were classified according to the National Cancer Institute Common Terminology Criteria. Review computed tomography was conducted 1,3 and 6 months after GSM-TACE treatment in order to determine the occurrence of re-bleeding; the tumor response was evaluated based on the Modified Response Evaluation Criteria In Solid Tumors and the expression levels of $\alpha$-feroprotein. The patients were followed-up for 1-6 months (average, 5.15 \pm 1.67 months). Following GSM-TACE treatment, 13 cases reached successful hemostasis without technical complications. The survival rates 1,3 and 6 months after treatment were 76.9 (10/13), 61.5 $(8 / 13)$ and $53.8 \%(7 / 13)$, respectively; the objective response rates were $61.6,53.9$ and $38.5 \%$, respectively. The primary post-operative complications were pain (100\%), nausea and vomiting (69.2\%), and fever (53.8\%). Among the 13 patients, 2 cases underwent surgical excision 10 and 30 days after GSM-TACE, and 1 case experienced re-bleeding 3 months after treatment, after which the patient received a second treatment with TACE and successful achieved hemostasis.
\end{abstract}

Correspondence to: Professor Yue Wei Zhang, Department of Interventional Therapy, Affiliated Zhongshan Hospital of Dalian University, 6 Jie Fang Street, Dalian, Liaoning 116001, P.R. China E-mail: yueweizhangdr@126.com

Key words: hepatocellular carcinoma, gelatin sponge microparticles, spontaneous rupture, hepatectomy, embolization, prognosis, survival, therapeutic
In conclusion, GSM-TACE of various diameters is a safe and effective method in the treatment of spontaneous rupture of hepatocellular carcinoma hemorrhage. GSM-TACE is able to achieve immediate hemostasis and improves the survival rate of patients, thus creating favorable conditions for follow-up treatment.

\section{Introduction}

Hepatocellular carcinoma (HCC), also known as liver cancer, is the sixth most common malignant tumor worldwide. Each year, 748,000 patients are diagnosed with liver cancer, and $\sim 696,000$ of these succumb to the disease; liver cancer accounts for $\leq 1 / 3$ of various malignant tumors. In China, the number of patients with liver cancer is $>50 \%$ larger than the total number of patients that develop liver cancer worldwide (1). Spontaneous rupture of HCC hemorrhage is a severe complication of HCC; the incidence of spontaneous rupture in Western countries is $\sim 3 \%$, and the mortality rate in Asian countries is $12-14 \%$ (2). As a result of its acute onset, the spontaneous rupture of HCC hemorrhages often leads to hemorrhagic shock, and if timely and reasonable treatment methods are not provided, the lives of the patients are put at risk (3-7).

Surgical resection, transarterial chemoembolization (TACE) and transcatheter arterial embolization (TAE) are common methods used in the treatment of spontaneous rupture of HCC hemorrhage (8-10). Recently, a novel method combining surgery and radio frequency ablation was reported for the treatment of spontaneous rupture of HCC hemorrhage (11). However, despite the presence of numerous positive treatment methods, a large number of patients are not successfully treated, and the mortality rate within 30 days is as high as $31-67 \%(8,12-15)$. Common embolic agents currently used in the clinical treatment of acute spontaneous rupture of HCC hemorrhage by TACE or TAE are iodized oil, gelatin sponge particles (Gelfoam), stainless steel rings and polyvinyl alcohol (16,17); to date, gelatin sponge microparticles (GSMs) combined with chemotherapy for the treatment of this disease has not been reported.

Embolization materials can be classified into three groups by the duration of embolization effect: Short-term, medium-term, and long-term. GSMs are medium-term embolic agents produced in China. A previous study using animal experimentation, performed at the Affiliated Zhongshan Hospital of 
Dalian University (Dalian, China), demonstrated the safety of GSMs (18). Clinical application studies have suggested that GSM-TACE in the treatment of HCC results in tumor necrosis and a reduction in tumor size, and has good clinical curative effects (19-21). The present study aimed to summarize the clinical hemostatic effect of GSM-TACE in the treatment of HCC, and to investigate the safety and efficacy of the clinical application of GSMs.

\section{Materials and methods}

Clinical materials. Data from a total of 13 cases of HCC combined with spontaneous rupture of HCC hemorrhage, who were treated between August 2010 and June 2014, were collected at the Affiliated Zhongshan Hospital of Dalian University (Dalian, China). The 13 patients were admitted to hospital $1 \mathrm{~h}$ to 2 days after suddenly developing acute abdominal pain or liver region pain, and 8 patients exhibited signs of peritoneal irritation. Imaging examinations, including computed tomography (CT), B ultrasound and magnetic resonance imaging, combined with the recording of clinical manifestations or diagnostic abdominal puncture, confirmed that the patients had a rupture of HCC (Table I). Baseline liver enzymes, including aspartate transaminase (AST), alanine aminotransferase (ALT), total bilirubin (TBIL) and albumin (ALB), were obtained prior to the GSM-TACE. The present study was approved by the Ethical Committee of the Affiliated Zhongshan Hospital of Dalian University (Dalian, China). Written informed consent was obtained from the patients or the patient's family.

GSM-TACE technique. The Seldinger method (22) was used to puncture the right femoral artery, a 5F-RH catheter (Terumo, Tokyo, Japan) was implanted into the hepatic duct, and routine celiac artery and hepatic artery angiography was subsequently performed (22). Heterotopic feeding artery angiography, including the superior mesenteric artery, phrenic artery, right renal artery and left gastric artery angiography, was conducted according to tumor location, size, tumor staining integrity and contrast extravasation, in order to confirm the tumor feeding arteries. Digital subtraction angiography (DSA) was performed to map the vascular liver anatomy to determine the presence of tumor staining and to identify the arterial feeders of the tumor. Tumor stains were specific findings of the HCC in DSA. The operational approach employed, GSM-TACE, was similar to routine TACE technique (22), aside from the use of GSMs [Hangzhou Aili Kang Pharmaceutical Technology Co, Ltd. (Hangzhou, China); size, $100 \mathrm{mg}$ ] with various diameters (350-560, 560-710 and 710-1,000 $\mu \mathrm{m})$ and dosages $(30-150 \mathrm{mg})$, according to tumor size and intraoperative blood flow velocity. GSMs were mixed with $10 \mathrm{mg}$ lobaplatin injection (Hainan Chang'an International Pharmaceutical Co., Ltd., Chang'an, China; $10 \mathrm{mg}$ ) to make the particle suspension. The GSMs and chemotherapeutic drug suspension were slowly infused via the transcatheter into the feeding artery in the tumor region, and the infusion was continued until the regional intra-arterial blood stagnation and tumor staining disappeared completely. Intraoperative angiography can be used to determine whether embolization should be performed. Following thoroughly embolization, the catheter was placed in the celiac artery, and once arteriography revealed the splenic artery, liver protection therapy was performed; $15 \mathrm{ml}$ polyene phosphatidylcholine injection and $1.8 \mathrm{~g}$ glutathione (Fudan Fuhua Pharmaceutical Co., Ltd., Shanghai, China) were mixed with $150 \mathrm{ml}$ glucose to a concentration of $5 \%$, and slowly infused.

Post-operative management. Patients were fasted for 1-2 days and remained on bed rest. Fluid infusion with saline and glucose was performed for 3-5 days (1,500-2,500 ml/day). In addition, the patients received the following: Intravenous injection of $2 \mathrm{~g}$ cefazolin sodium (Le Pu Pharmaceutical Co., Ltd., Henan, China), twice daily; intravenous injection of coenzyme complex $1+100 \mathrm{ml}$ glucose with a concentration of 5\% (Shuanglu Pharmaceutical Co., Ltd., Beijing, China), once daily; intravenous injection of $15 \mathrm{ml}$ polyene phosphatidylcholine injection $+100 \mathrm{ml}$ glucose with a concentration of 5\% (Tiantai Mountain Pharmaceutical Co., Ltd., Sichuan, China), once daily; intravenous injection of $1.8 \mathrm{~g}$ glutathione $+100 \mathrm{ml}$ sodium chloride with a concentration of $0.9 \%$, once daily; intravenous injection of $40 \mathrm{mg}$ esomeprazole (AstraZeneca Pharmaceutical Co., Ltd., Jiangsu, China) $+0.9 \%$ sodium chloride $100 \mathrm{ml}$, once daily; intravenous injection of $5 \mathrm{mg}$ tropisetron (Yikang Pharmaceutical Co., Ltd., Shandong, China) + 0.9\% sodium chloride $100 \mathrm{ml}$, once daily; intramuscular injection of $1 \mathrm{KU}$ hemocoagulase (Aohong Pharmaceutical Co., Ltd., Liaoning, China), once daily; and intramuscular injection of $10 \mathrm{mg}$ vitamin K1 (Yikang Pharmaceutical Co., Ltd., Shandong, China), once daily. Post-operative fever occurred in seven patients, and symptomatic treatment [compound aminophenazone (Shuanghe Pharmaceutical Co., Ltd., Shanxi, China)] was administered via intramuscular injection when the temperature of a patient was $>38.5^{\circ} \mathrm{C}$. Post-operatively, all patients had right upper abdominal pain, and were treated according to WHO analgesic three principles (23).

Evaluation of the curative effects and observation of adverse effects. Evaluation was performed based on the Response Evaluation Criteria In Solid Tumors (mRECIST 1.1) (24). Target lesions were scored as follows: Complete response (CR), all target lesions developed during the arterial enhancement period ceased to exist, and all lymph nodes (pathological) were $<10 \mathrm{~mm}$; partial response (PR), total target lesion arterial enhancement length diameter was reduced $\geq 30 \%$; progressive disease (PD), total baseline lesion diameter was increased $\geq 20 \%$, or new lesions occured; and stable disease (SD), total baseline lesion diameter was reduced but did not reach the level of PR, or the level was increased but not as high as in PD.

Non-target lesions were scored as follows: CR, all non-target lesions developed during the arterial phase ceased to exist and the tumor marker levels were normal (normal AFP, $0-20 \mathrm{ng} / \mathrm{ml}$ ); SD, $\geq 1$ non-target lesions developed during the arterial phase remained, or tumor marker levels were higher than normal levels; and PD, the appearance of $\geq 1$ new lesions and/or the existence of non-target lesions. The tumor objective response rate $(\mathrm{ORR}$; ORR $=\mathrm{CR}+\mathrm{PR} /$ total number of patients in each time segment) of each time segment was calculated. The time segment was the time from the initial GSM-TACE to the evaluation of the curative effects. At 4 days after surgery, a review $\mathrm{CT}$ scan was performed to evaluate 
the hemostasis effect and tumor necrosis degree following GSM-TACE. Routine blood, liver and kidney function tests, and $\alpha$-fetoprotein (AFP) examination, were conducted at 4 and 7 days and every month after surgery. An enhanced $\mathrm{CT}$ examination was performed every month after surgery in order to evaluate whether there was re-bleeding or new lesions; if there was no re-bleeding, subsequent GSM-TACE treatment was performed to control tumor growth. The purpose of the treatment was to prolong the survival of patients.

Statistical analysis. Statistical analyses were performed using SPSS 17.0 software (SPSS, Inc., Chicago, IL, USA). P<0.05 was considered to indicate a statistically significant difference.

\section{Results}

Patient characteristics. Among the 13 cases of patients with HCC, 11 cases had tumors located in the right lobe and 2 cases had tumors located in the left lobe. A total of 11 cases had tumors protruding from the surface of the liver capsule, and various degrees of blood accumulation could be observed in the inferior liver capsule and abdominal cavity (Fig. 1A). Baseline liver enzymes were obtained prior to the GSM-TACE

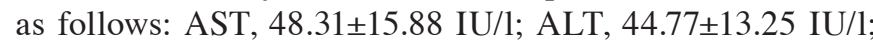
TBIL, 24.46 $\pm 5.53 \mu \mathrm{mol} / \mathrm{l}$; and ALB, 39.69 $\pm 4.36 \mathrm{~g} / \mathrm{l}$. Prior to GSM-TACE, DSA showed that all 13 cases had hypervascular tumors with strong tumor staining (Fig. 1B). Following GSM-TACE surgery, all 13 cases in the group achieved successful hemostasis, and the tumor staining disappeared (Fig. 1C). At 4 days after GSMs-TACE, a review CT scan was conducted and the results showed that 13 cases had no significant increase in hepatic subcapsular hematoma and hemoperitoneum, and different degrees of 'honeycomb' necrosis could be seen in lesions (Fig. 1D). Among the 13 cases, review CT showed that 2 cases had tumors located in the liver $\mathrm{V}$ segment and VI segment edge with significant necrosis, and no re-bleeding. These 2 cases received selective tumor resection at 10 days post-operation and 1 month post-operation, respectively. During the surgery, the tumor invaded the liver capsule which resulted in tumor ulceration, and obvious necrosis tissues could be observed. One case had a re-rupture of the hepatocellular carcinoma hemorrhage at 3 months after surgery, and received a second GSM-TACE for successful hemostasis. All the cases had a review CT scan at 1 month after surgery, and these showed that the intrahepatic tumor size of 7 cases $(53.8 \%)$ was reduced compared with baseline lesions (Fig. 1E). Post-operative pathological examination confirmed that the tumor was a moderately differentiated hepatocellular carcinoma (Fig. 2).

Adverse reactions following GSM-TACE. Patients had various degrees of right upper abdominal pain and nausea and vomiting following surgery, and this may be related to tumor rupture and reactions following embolism. Following surgery, 7 patients experienced symptoms of fever, and body temperature fluctuated of $37.1-38.9^{\circ} \mathrm{C}$. Liver enzymes were measured four days after the GSM-TACE as follows: AST, 60.85 $\pm 13.53 \mathrm{IU} / \mathrm{l}(\mathrm{P}=0.037)$; ALT, 54.23 $\pm 12.72 \mathrm{IU} / \mathrm{l}(\mathrm{P}=0.001)$; TBIL, 38.77 $\pm 4.67 \mu \mathrm{mol} / \mathrm{l}$ $(\mathrm{P}=0.011)$; and ALB, 37.77 $\pm 3.61 \mathrm{~g} / \mathrm{l}(\mathrm{P}=0.001)$. Although these results were significantly increased and indicative of mild impaired liver function, this was only transient and liver function
Table I. General information of the patients.

\begin{tabular}{|c|c|}
\hline Parameter & $\mathrm{n}$ \\
\hline Age range (mean) & $43-77(58.07 \pm 10.41)$ \\
\hline \multicolumn{2}{|l|}{ Gender } \\
\hline Male & 13 \\
\hline Female & 0 \\
\hline \multicolumn{2}{|l|}{ Etiology } \\
\hline Hepatitis B & 12 \\
\hline Hepatitis C & 1 \\
\hline Child-Pugh grading, A/B & $9 / 4$ \\
\hline ECOG score, $0 / 1 / 2$ & $12 / 1 / 0$ \\
\hline BCLC stage, $\mathrm{A} / \mathrm{B} / \mathrm{C} / \mathrm{D}$ & $0 / 13 / 0 / 0$ \\
\hline Tumor size range, $\mathrm{cm}$ (mean) & $4.2-10(6.21 \pm 2.18)$ \\
\hline$<5 \mathrm{~cm}$ & 2 \\
\hline$\geq 5 \mathrm{~cm}$ & 11 \\
\hline Tumor number, $<2 />2$ & $9 / 4$ \\
\hline Tumor light out liver surface & 11 \\
\hline $\mathrm{AFP},<400 />400 \mathrm{ng} / \mathrm{ml}$ & $9 / 4$ \\
\hline GSM-TACE cycles, 1/2-3/>3 & $5 / 8 / 0$ \\
\hline
\end{tabular}

ECOG, Eastern Cooperative Oncology Group; BCLC, Barcelona clinic liver cancer; AFP, $\alpha$-fetoprotein; GSM-TACE, gelatin sponge microparticle-transcatheter arterial chemoembolization.

Table II. Adverse reactions and complications following gelatin sponge microparticle-transcatheter arterial chemoembolization treatment.

\begin{tabular}{lc}
\hline Complications & $\mathrm{n}(\%)$ \\
\hline I-II degree & \\
Upper abdominal pain & $13(100.0)$ \\
Nausea and vomiting & $9(69.2)$ \\
Fever & $7(53.8)$ \\
Neutropenia & $0(0.0)$ \\
III degree & \\
Cholecystitis & $0(0.0)$ \\
Liver abscess & $0(0.0)$ \\
Tumor recurrent rupture and bleeding & $1(7.6)$ \\
Hepatic failure & $1(7.6)$ \\
Renal failure & $1(7.6)$ \\
Hepatic encephalopathy & $1(7.6)$ \\
Upper gastrointestinal bleeding & $1(7.6)$ \\
\hline
\end{tabular}

indices recovered to the normal levels (AST, 37.51 $\pm 13.84 \mathrm{IU} / \mathrm{l}$; ALT, 35.47 $\pm 12.41 \mathrm{IU} / \mathrm{l}$; TBIL, 21.86 $\pm 3.84 \mu \mathrm{mol} / \mathrm{l}$; and ALB, $43.77 \pm 11.38 \mathrm{~g} / \mathrm{l})$ after $\sim 7$ days of supportive liver protection therapy. A total of 13 cases had I-II degree adverse reactions following surgery (Table II).

Therapeutic evaluation. The survival rates at 1,3 and 6 months after surgery were $76.9(10 / 13), 61.5(8 / 13)$ and 
A

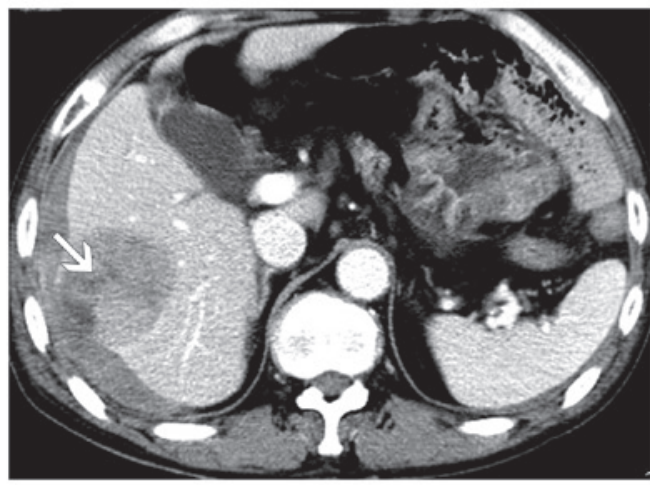

$\mathbf{C}$

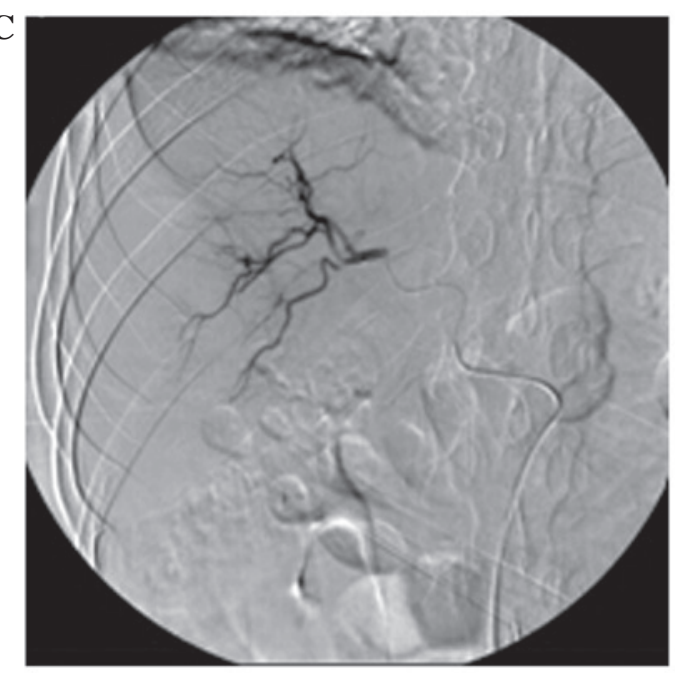

B

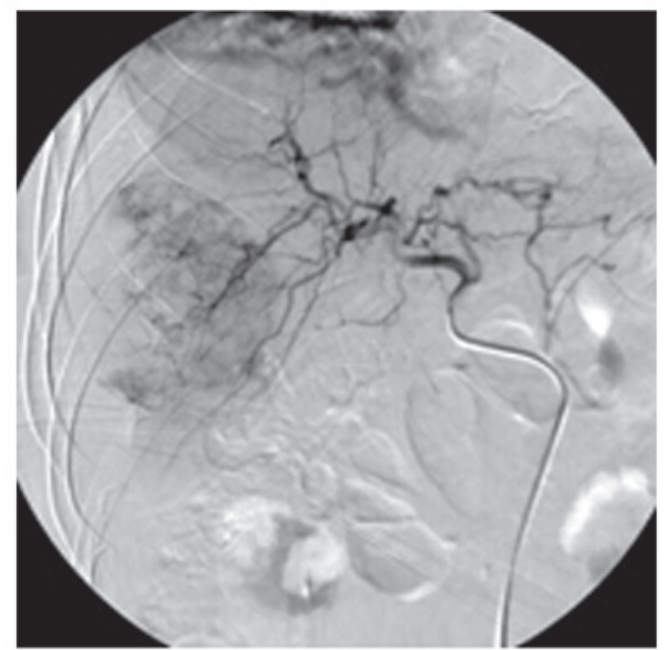

D

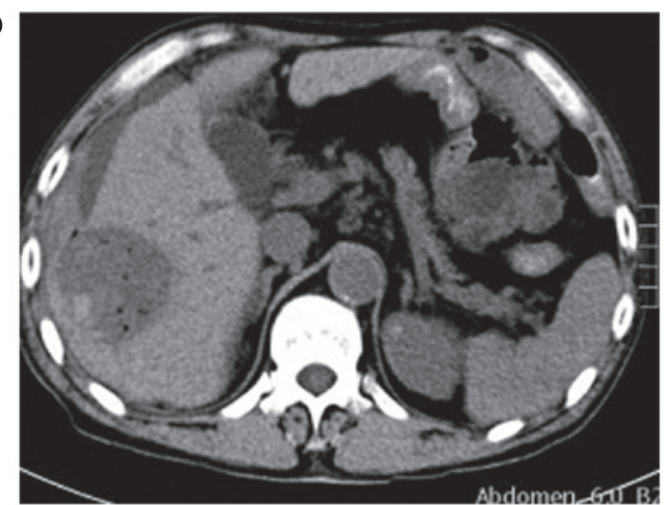

$\mathbf{E}$

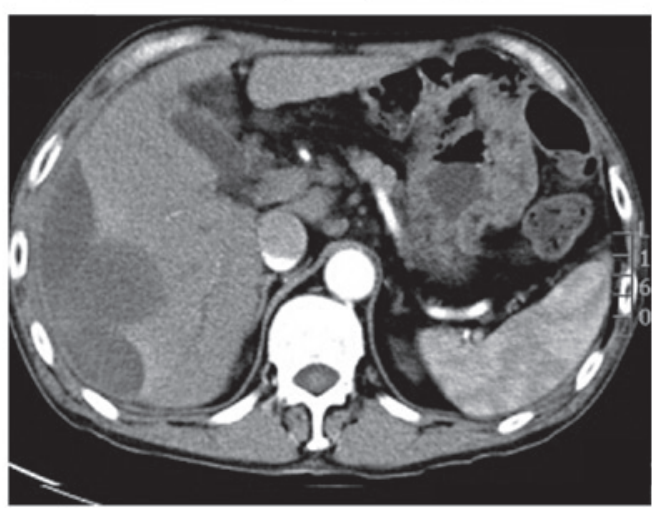

Figure 1. (A) Enhanced computed tomography (CT) prior to gelatin sponge microparticle-transcatheter arterial chemoembolization (GSM-TACE) showed that the tumor extrudes the liver capsule, and hepatic subcapsular hematoma, intra-tumoral liquefied area and rupture could be observed (white arrow). (B) digital subtraction angiography in GSM-TACE showed right hepatic lobe tumor staining. (C) Following GSM-TACE treatment, the artery of the right hepatic lobe tumor was blocked and tumor staining disappeared, suggesting successful hemostasis. (D) A review CT scan at 4 days after GSM-TACE showed 'honeycomb-like' low density necrosis in the lesion; hepatic subcapsular hemorrhage was not increased. (E) A review enhanced CT at 1 month after GSM-TACE showed no fresh hepatic subcapsular hemorrhage, no enhanced lesions could be observed in the original lesion arterial phase, and tumor size was markedly reduced.

$53.8 \%$ (7/13), respectively. Until June 2014, the follow-up time was 3 years and 10 months, during which 4 cases did not survive $(30.7 \%)$, including 1 case who succumbed to hepatic encephalopathy, 2 cases who succumbed to renal failure and 1 case who succumbed to upper gastrointestinal hemorrhage (Table III).

\section{Discussion}

Interventional embolization is the first choice of emergency treatment for HCC rupture hemorrhage $(25,26)$. A previous meta-analysis (16) revealed that the success rate of TAE hemostatic therapy for the treatment of HCC rupture hemorrhage in the acute stage was $53-100 \%$ (16); the 30-day mortality of TAE treatment (0-37\%) was lower than that of surgical resection $(28-75 \%)$, suggesting that regardless of whether patients with HCC have bleeding or not, the survival rate will be similar following treatment (16). Kirikoshi et al (14) demonstrated that the post-operative survival rate of TAE treatment was significantly higher compared with surgical resection; mortality rates following surgical resection within 30 days of surgery were $30-70 \%$, and the mortality rates following 
Table III. Tumor response after gelatin sponge microparticle-transcatheter arterial chemoembolization treatment.

\begin{tabular}{lcccccr}
\hline Months after surgery & $\mathrm{n}$ & Complete response $^{\mathrm{a}}$ & Partial response $^{\mathrm{a}}$ & Stable disease $^{\mathrm{a}}$ & Progressive disease $^{\mathrm{a}}$ & ORR $^{\mathrm{a}}$ \\
\hline 1 & 12 & $3(23.1)$ & $5(38.5)$ & $2(15.4)$ & $2(15.4)$ & $8(61.6)$ \\
3 & 10 & $2(15.4)$ & $5(38.5)$ & $2(15.4)$ & $1(7.6)$ & $7(53.9)$ \\
6 & 9 & $2(15.4)$ & $3(23.1)$ & $2(15.4)$ & $2(15.4)$ & $5(38.5)$ \\
\hline
\end{tabular}

${ }^{a} \mathrm{n}(\%)$. ORR, objective response rate.

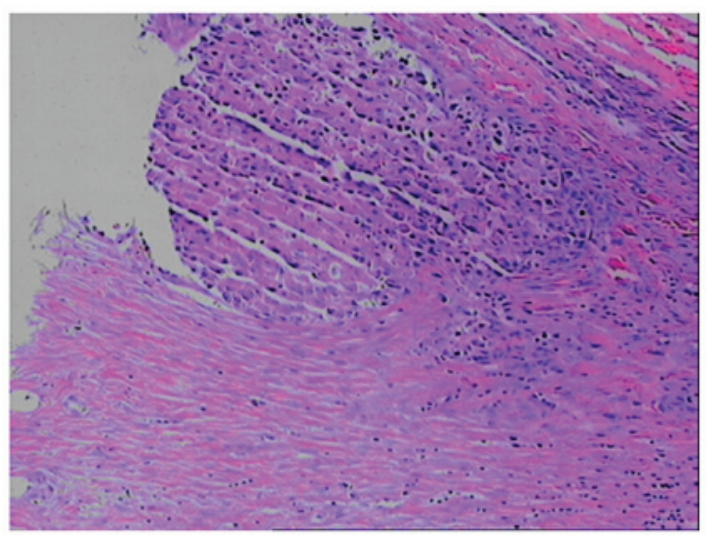

Figure 2. Pathological section following surgical resection and gelatin sponge microparticle-transcatheter arterial chemoembolization (magnification, x100).

TAE treatment within 30 days of surgery were $<10 \%$. Tumor resection following TACE or TAE for the treatment of patients with HCC greatly increased the survival rate of the patients (27). The long-term outcomes of surgical resection were improved compared with TACE treatment for $\mathrm{HCC}$, and resection was able to remove the tumor completely (28-30). All 13 patients with rupture of hepatocellular carcinoma hemorrhage received GSM-TACE treatment between $1 \mathrm{~h}$ and 2 days after bleeding; among them, 2 cases received surgical resection following GSM-TACE treatment. After follow-up between 4 and 6 months, the results demonstrated that no recurrence or metastatic lesions were observed among the patients who received surgical resection. It is believed that TACE is preferable in the treatment of spontaneous rupture of hepatocellular carcinoma hemorrhage, and elective surgical resection may help to improve the long-term curative effect.

Tumor rupture hemorrhage is one of the severe complications of primary hepatocellular carcinoma, and the underlying mechanisms remain unclear. A number of investigators believe that tumor invasion in hepatic veins may cause congestion, and increased tumor growth speed could cause central necrosis combined with coagulation disorders, and the tumor tissue edge is vulnerable, so trauma or a sudden increase in intra-abdominal pressure and the friction between the diaphragmatic muscle and the tumor, caused by respiratory motion could result in rupture and bleeding $(26,31)$. In the present study, 11 cases had a tumor protruding from the liver surface, and 9 cases had a tumor protruding from the liver surface $>1 \mathrm{~cm}$. The results of the current study demonstrate that tumor size $>5 \mathrm{~cm}$ and tumors protruding from the liver surface are one of the primary risk factors of $\mathrm{HCC}$ rupture and bleeding. In the present study, 1 case had a rupture of HCC hemorrhage, and no follow-up was conducted following emergency GSM-TACE embolization; after 3 months, re-bleeding occurred and hemostasis was achieved after treatment with the same embolization therapy. To date, the patient is in a stable condition according to mRECIST; stable disease and the diameter of the tumor were reduced but a partial response was not achieved. Bleeding following GSM-TACE treatment may be associated with the following factors: i) The tumor protruding into the liver surface; ii) excessive embolism therapy dosages; iii) a large tumor with internal liquefaction necrosis and increasing pressure; and iv) tumor progression. In the present study, the patients underwent hemostasis following GSM-TACE treatment, and the tumor growth was effectively inhibited during follow-up the period.

In the current study, the clinical characteristic of TACE in the treatment of HCC rupture and bleeding were assessed following the application of particulate embolic agent-GSM in regional tumor arterial embolization. Worldwide, embolic agents for the treatment of HCC rupture and bleeding typically include iodized oil, gelatin sponge particles, polyvinyl alcohol and stainless steel ring $(17,29)$, and each agent has its advantages and disadvantages. The agents using iodized oil as the primary embolic agent after years of clinical precipitation are used widely, but during the treatment of HCC by TACE, the necrotic rate of tumors is only $0-4.8 \%(32,33)$, and it may lead to severe complications, such as pulmonary embolism (34). However, the application of iodized oil embolization agent is considered to be one of the factors that cause tumor rupture $(35,36)$.

The application of permanent embolic agents and steel rings is not conducive to the future treatment of HCC. The GSMs used in the present study were particle-type mid-embolic agents made in China, and preliminary animal experiments and clinical studies have confirmed their safety and efficacy, which is suitable for the treatment of large HCCs and combined arteriovenous fistula and portal vein thrombosis; in the present study, tumor necrosis could be observed at $3 \mathrm{~h}$ after surgery with few complications $(18,19)$. In addition, in the current study, no re-bleeding occurred in 13 cases $(100 \%) 1$ month after GSM-TACE. Grade I-II adverse reactions, such as fever, right upper abdominal pain, nausea and vomiting, are common complications following GSM-TACE (37); in the present study, the liver and kidney functions were recovered to the pre-operative levels 7-10 days after surgery, tumor growth was effectively controlled, and the ORR at 1, 3 and 6 months after surgery was 61.6, 53.9 and 
$38.5 \%$, respectively, leading to the conclusion that the survival rate of the patients was prolonged. The occurrence of the above-mentioned complications may be associated with tumor necrosis. In the current study, 4 cases succumbed to complications, such as tumor progression complicated with multiple organ failure, gastrointestinal bleeding and hepatic encephalopathy; however, patient mortality showed no direct correlation with GSM-TACE. The results of the present study were concordant with those of previous studies with regards to the safety and clinical efficacy of GSMs for the treatment of HCC (19-21).

Pre-operative shock, low hemoglobin and albumin expression levels, prolonged prothrombin time, increased serum creatinine expression levels, hepatic encephalopathy, severe ascites, portal vein tumor thrombus and multinodular tumors in the left and right lobe are the primary factors that affect the treatment of HCC rupture hemorrhage. Liver function is one of the prognostic factors for survival time in TAE and TACE (25). According to the Barcelona clinic liver cancer (BCLC) standard, all of the patients in the present study were suitable for GSM-TACE treatment. During surgery, the dosage was personalized for each individual according to patients' conditions, as suitable quantity of embolism is a key factor in achieving good clinical efficacy. In addition, the protection of liver function during and following TACE is important.

In conclusion, in patients with acute $\mathrm{HCC}$ rupture, bleeding with stable vital signs, BCLC B stage and Child A-B grade can be treated with TACE as a first line of treatment. After reaching a stable condition, treatment with surgery can be conducted appropriately according to lesion location, size, number, metastasis status and tumor progression, which is conducive to improve the long-term curative effect. GSM-TAC for the treatment of HCC rupture and bleeding has the advantages of a good hemostatic effect, and good safety and short-term effects; however, due to the small sample size used in the current study, the long-term effects need to be verified by investigating a larger sample size.

\section{References}

1. Jemal A, Bray F, Center MM, Ferlay J, Ward E and Forman D: Global cancer statistics. CA Cancer J Clin 61: 69-90, 2011.

2. Battula N, Madanur M, Priest O, Srinivasan P, O'Grady J, Heneghan MA, Bowles M, Muiesan P, Heaton N and Rela M: Spontaneous rupture of hepatocellular carcinoma: A Western experience. Am J Surg 197: 164-167, 2009.

3. Okazaki M, Higashihara H, Koganemaru F, Nakamura T, Kitsuki H, Hoashi $\mathrm{T}$ and Makuuchi $\mathrm{M}$ : Intraperitoneal hemorrhage from hepatocellular carcinoma: Emergency chemoembolization or embolization. Radiology 180: 647-651, 1991.

4. Sato Y, Fujiwara K, Furui S, Ogata I, Oka Y, Hayashi S, Ohta Y, Iio $\mathrm{M}$ and $\mathrm{Oka} \mathrm{H}$ : Benefit of transcatheter arterial embolization for ruptured hepatocellular carcinoma complicating liver cirrhosis. Gastroenterology 89: 157-159, 1985.

5. Corr P, Chan M, Lau WY and Metreweli C: The role of hepatic arterial embolization in the management of ruptured hepatocellular carcinoma. Clin Radiol 48: 163-165, 1993.

6. Xu HS and Yan JB: Conservative management of spontaneous ruptured hepatocellular carcinoma. Am Surg 60: 629-633, 1994.

7. Hsieh JS, Huang CJ, Huang YS, Sheen PC and Huang TJ: Intraperitoneal hemorrhage due to spontaneous rupture of hepatocellular carcinoma: Treatment by hepatic artery embolization. AJR Am J Roentgenol 149: 715-717, 1987.

8. Li WH, Cheuk EC, Kowk PC and Cheung MT: Survival after transarterial embolization for spontaneous ruptured hepatocellular carcinoma. J Hepatobihary Pancreat Surg 16: 508-512, 2009.
9. Yang T, Sun YF, Zhang J, Lau WY, Lai EC, Lu JH, Shen F and Wu MC: Partial hepatectomy for ruptured hepatocellular carcinoma. Br J Surg 100: 1071-1079, 2013.

10. Yeh CN, Lee WC, Jeng LB, Chen MF and Yu MC: Spontaneous tumour rupture and prognosis in patients with hepatocellular carcinoma. Br J Surg 89: 1125-1129, 2002.

11. Cheung TT, Poon RT, Chok KS, Chan AC, Tsang SH, Dai WC, Yau TC, Chan SC, Fan ST and Lo CM: Management of spontaneously ruptured hepatocellular carcinomas in the radiofrequency ablation era. PLoS One 9: e94453, 2014.

12. Toshikuni N, Takuma Y, Morimoto Y, Shimomura H and Yamamoto $\mathrm{H}$ : Transarterial embolization for ruptured hepatocellular carcinoma: Survival predictors. Hepatogastroenterology 58: 565-569, 2011.

13. Tan FL, Tan YM, Chung AY, Cheow PC, Chow PK and Ooi LL: Factors affecting early mortality in spontaneous rupture of hepatocellular carcinoma. ANZ J Surg 76: 448-452, 2006.

14. Kirikoshi H, Saito S, Yoneda M, Fujita K, Mawatari H, Uchiyama $T$, Higurashi $T$, Imajo $K$, Sakaguchi $T$, Atsukawa K, et al: Outcomes and factors influencing survival in cirrhotic cases with spontaneous rupture of hepatocellular carcinoma: A multicenter study. BMC Gastroenterol 9: 29, 2009.

15. Kung CT, Liu BM, Ng SH, Lee TY, Cheng YF, Chen MC and Ko SF: Transcatheter arterial embolization in the emergency department for hemodynamic instability due to ruptured hepatocellular carcinoma: Analysis of 167 cases. Am J Roentgenol 191: W231-W239, 2008.

16. Lai EC and Lau WY: Spontaneous rupture of hepatocellular carcinoma: A systematic review. Acta Radiol 141: 191-198, 2006.

17. Lau WY: Primary hepatocellular carcinoma. In: Disease of the Liver and Biliary Tract. Blumgart LH and Fong Y (eds). 3rd edition. WB Saunders Co, Ltd., London, England, pp1423-1450, 2000.

18. Zhang YW, Ao J, Liu Y, Qiao MX, Yang XL, Tang SX, Li C and $\mathrm{Xu} \mathrm{K}$ : Pharmacokinetics of gelatin sponge microparticles in a rabbit VX2 liver tumor model of hepatic arterial chemoembolization. Tumour Biol 35: 10905-10910, 2014.

19. Zhang YW and Liu Y: Transcatheter arterial chemoembolization of hepatocellular carcinoma with 350-560 $\mu \mathrm{m}$ gelatin sponge particles: Efficacy, tumour response and survival. Zhonghua Gan Zang Bing Za Zhi 21: 637-638, 2013 (In Chinese).

20. Kamran AU, Liu Y, Li FE, Liu S, Wu JL and Zhang YW: Transcatheter arterial chemoembolization with gelatin sponge microparticles treated for BCLC stage B hepatocellular carcinoma: A single center retrospective study. Medicine (Baltimore) 94: e2154, 2015.

21. Liu Y, Zhang Y, Bautista D, Tang S, Zhou J, Li C and Zhao G: Trans-arterial p53-gene-embolization with gelatin sponge microparticles for hepatocellular carcinoma with BCLC stage B: Single-centre experience. Cell Biochem Biophys 71: 99-104, 2015.

22. Ramsey DE, Kernagis LY, Soulen MC and Geschwind JF: Chemoembolization of hepatocellular carcinoma . J Vasc Intery Radiol 13: S211-S221, 2002.

23. World Health Organization: Cancer Pain Relief and Palliative Care: Report of a WHO Expert Committee. Geneva, 1990, pp7-21.

24. Eisenhauer EA, Therasse P, Bogaerts J, Schwartz LH, Sargent D, Ford R, Dancey J, Arbuck S, Gwyther S, Mooney M, et al: New response evaluation criteria in solid tumours: revised RECIST guideline (version 1.1). Eur J Cancer 45: 228-247, 2009.

25. Shin BS, Park MH and Jeon GS: Outcome and prognostic factors of spontaneous ruptured hepatocellular carcinoma treated with transarterial embolization. Acta Radiol 52: 331-335, 2011.

26. Zhu LX, Wang GS and Fan ST: Spontaneous rupture of hepatocellular carcinoma. Br J Surg 83: 602-607, 1996.

27. Jin YJ, Lee JW, Park SW, Lee JI, Lee DH, Kim YS, Cho SG, Jeon YS, Lee KY and Ahn SI: Survival outcome of patients with spontaneously ruptured hepatocellular carcinoma treated surgically or by transarterial embolization. World J Gastroenterol 19: 4537-4544, 2013.

28. Liu CL, Fan ST, Lo CM, Tso WK, Poon RT, Lam CM and Wong J: Management of spontaneous rupture of hepatocellular carcinoma: Single-center experience. J Clin Oncol 19: 3725-3732, 2001.

29. Tarantino L, Sordelli I, Calise F, Ripa C, Perrotta M and Sperlongano P: Prognosis of patients with spontaneous rupture of hepatocellular carcinoma in cirrhosis. Updates Surg 63: 25-30, 2011.

30. Fujii M, Miyake H, Takamura K and Tashiro S: Management of spontaneous ruptured hepatocellular carcinoma. Nihon Geka Gakkai Zasshi 105: 292-295, 2004 (In Japanese). 
31. Tanaka T, Yamanaka N, Oriyama T, Furukawa K and Okamoto E: Factors regulating tumor pressure in hepatocellular carcinoma and implications for tumor spread. Hepatology 26: 283-287, 1997.

32. Llovet JM and Bruix J: Systematic review of randomized trials for unresectable hepatocellular carcinoma: Chemoembolization improves survival. Hepatology 37: 429-442, 2003.

33. Cammà $\mathrm{C}$, Schepis $\mathrm{F}$, Orlando $\mathrm{A}$, Albanese $\mathrm{M}$, Shahied L, Trevisani F, Andreone P, Craxì A and Cottone M: Transarterial chemoembolization for unresectable hepatocellular carcinoma: Meta-analysis of randomized controlled trials. Radiology 224: 47-54, 2002.

34. Kim YJ, Lee HG, Park JM, Lim YS, Chung MH, Sung MS, Yoo WJ and Lim HW: Polyvinyl alcohol embolization adjuvant to oily chemoembolization in advanced hepatocellular carcinoma with arterioportal shunts. Korean J Radiol 8: 311-319, 2007.
35. Miyoshi A, Kitahara K, Kohya N, Noshiro H and Miyazahi K: Outcomes of patients with spontaneous rupture of hepatocellular carcinoma. Hepatogastroenterology 58: 99-102, 2011.

36. Liu CL, Ngan H, Lo CM and Fan ST: Ruptured hepatocellular carcinoma as a complication of transarterial oily chemoembolization. Br J Surg 85: 512-514, 1998.

37. National Cancer Institute. Common Terminology Criteria for Adverse Events (CTCAE) version 4. United States Department of Health and Human Services, 2009. Available at: http://evs.nci.nih. gov/ftp1/CTCAE/CTCAE_4.03_2010-06-14_Quick Reference_5x7 pdf. Accessed February 22, 2012. 